\title{
Metal-Insulator transition in Hubbard-like models with random hopping
}

\author{
Matthew S. Foster* and Andreas W. W. Ludwig \\ Department of Physics, University of California, Santa Barbara, CA 93106
}

(Dated: September 20, 2018)

\begin{abstract}
An instability of a diffusive Fermi liquid, indicative of a metal-insulator transition (expected to be of first order), arising solely from the competition between quenched disorder and shortranged interparticle interactions is identified in Hubbard-like models for spinless fermions, subject to (complex) random hopping at half-filling on bipartite lattices. The instability, found within a Finkel'stein non-linear sigma model treatment in $d=(2+\epsilon)>2$ dimensions, originates from an underlying particle-hole like (so-called chiral) symmetry, shared by both disorder and interactions. In the clean, interacting Fermi liquid this symmetry is responsible for the (completely different) nesting instability.
\end{abstract}

PACS numbers: $71.30 .+\mathrm{h}, 71.10 . \mathrm{Fd}, 72.15 . \mathrm{Rn}$

Understanding the combined effects of quenched disorder and interparticle interactions in electronic systems remains one of the central problems in solid state physics. Models of noninteracting electrons subject to static, random impurity potentials provide the simplest description of disordered metals; many analytical and numerical studies have shown that such models exhibit a continuous metal-insulator transition (MIT) in three dimensions (3D),$\frac{1}{=}$ accessed by varying either the disorder strength, or the Fermi energy relative to the mobility edge. By contrast, all electronic states are typically exponentially localized in one and two dimensions by arbitrarily weak disorder. (Notable exceptions occur in the presence of spin-orbit scattering, and in the systems discussed, e.g., in Refs. 2, 3. )

The above description ignores the important effects of interparticle interactions. Unfortunately, however, theories capturing the competition of both disorder and interactions are typically quite complex, and difficult to analyze reliably $\stackrel{4,5}{ \pm}$ These issues have come again to the forefront of scientific debate in view of discussions concerning a metal-insulator transition in $2 \mathrm{D}$, and related experimental results on $2 \mathrm{D}$ semiconductor inversion layers ${ }^{6}$

In the present work, we identify a novel "AndersonMott" instability of a diffusive Fermi liquid in $d=(2+\epsilon)>2$ spatial dimensions $(\epsilon \ll 1)$, which arises solely from the competition between disorder and short-range interactions. This instability is indicative of a metal-insulator transition (expected to be of first order) from the diffusive Fermi liquid to an insulating state dominated by both strong disorder and interactions. (See Fig. 1. below.) Since the system that we study has no localized phase with disorder in the absence of interactions, a localized phase can only appear due to the presence of the interactions. We expect our result to be relevant for sufficiently strong disorder in $d=3$ dimensions.

Specifically, we analyze a class of "Hubbard-like" models ${ }^{7}$ of spinless fermions, at half-filling on bipartite lattices, with random (short-range) hopping between the two sublattices. [The Hamiltonian is given below in Eq. (11).] In every realization of the (static) disorder, such a model possesses a special particle-hole like symmetry, which we will refer to as sublattice symmetry (SLS). [SLS is termed "chiral symmetry" in the classification scheme of Ref. 8 (see also Refs. 2, 3, $, 10,11,12,13$ ).]

In the absence of disorder, it is SLS which is responsible for the "nesting" condition of the Fermi surface. Fermi surface nesting is, in a sense, the defining property of (clean) Hubbard-like models for interacting lattice fermions in $d \geq 2$. It is the nesting condition which makes the ballistic Fermi liquid phase at half filling in such models unstable to Mott insulating order in the presence of generic, arbitrarily weak interparticle interactions $\underline{14,15,16}$ Here we consider, in addition, complex random (nearestneighbor) hopping, which breaks time reversal invariance (TRI) in every realization of disorder $\stackrel{17}{*}$ For our system of spinless fermions, this is consistent with the application of a random magnetic field to the otherwise clean model.

Our principal motivation for studying such a model is that we expect both disorder and interparticle interactions to play important roles in the description of the low-energy physics. Because random hopping preserves the special SLS, our disordered model retains the nesting instability of the associated clean system. This instability can therefore compete with the unusual localization physics of the disordered, but noninteracting model (see below). The further assumption of broken TRI guarantees that we do not have to confront an additional superconducting instability,$\frac{5,14,15,16}{}$ We expect the instability that we identify in this work in the simultaneous presence of both disorder and interactions to occur in three dimensions for sufficiently strong disorder, and we stress that it is clearly distinct from the pure Mott nesting instability, although the latter also appears in our model phase diagram (see Fig. 1, below). We note that the effects of hopping disorder upon the Néel ground state of the (slightly more complex) spin- $1 / 2$ Hubbard model at half filling were studied numerically in Ref. 18, although these studies were limited to $d=2$.

A second motivating factor is that, interestingly, the presence of SLS radically changes the localization physics of the disordered, noninteracting random hopping model [Eq. (11), below, with $V=U=0]$. SLS enables the ran- 
dom hopping model to evade the phenomenon of Anderson localization. Specifically, the noninteracting system exhibits a critical, delocalized phase at the band center (half filling) in one, two, and three dimensions for finite disorder strength, with a strongly divergent low-energy density of states in $d=1,2,2,3,9,10,11,12$ In particular, there is no MIT and no Anderson insulating phase in $d=3$ (in the absence of interactions). Random hopping models have been of significant theoretical interest in the recent past, both because of the unusual delocalization physics described above, but also because these models have proven amenable to a variety of powerful analytical techniques in $d \leq 2$, with many exact and/or nonperturbative features now understood: $2,10,12$ This situation should be contrasted with our understanding of the conventional noninteracting ("Wigner-Dyson") MIT, which is based largely on perturbative results in $d>2 . \frac{1}{1}$ Our work here addresses the effects of interparticle interactions in random hopping models for $d \geq 2$.

Our starting point is the following extended Hubbardlike Hamiltonian for spinless fermions hopping on a bipartite lattice at half filling:

$$
\begin{aligned}
H= & -\sum_{\langle i j\rangle}\left(t+\delta t_{i, j}\right) c_{A i}^{\dagger} c_{B j}+\text { H.c. }+2 V \sum_{\langle i j\rangle} \delta \hat{n}_{A i} \delta \hat{n}_{B j} \\
& +U\left(\sum_{\left\langle\left\langle i i^{\prime}\right\rangle\right\rangle} \delta \hat{n}_{A i} \delta \hat{n}_{A i^{\prime}}+\sum_{\left\langle\left\langle j j^{\prime}\right\rangle\right\rangle} \delta \hat{n}_{B j} \delta \hat{n}_{B j^{\prime}}\right) .
\end{aligned}
$$

Any bipartite lattice may be divided into two interpenetrating sublattices, which we distinguish with the labels $A$ and $B$. In Eq. (1), $c_{A i}^{\dagger}$ and $c_{B j}$ denote fermion creation and annihilation operators on the $A$ and $B$ sublattices, respectively. Here, $i$ and $j$ respectively index the $A$ and $B$ sublattice sites, and the sums on $\langle i j\rangle$ run over all nearest neighbor $A$ - $B$ lattice bonds, while the sums on $\left\langle\left\langle i i^{\prime}\right\rangle\right\rangle$ and $\left\langle\left\langle j j^{\prime}\right\rangle\right\rangle$ run over all next-nearest neighbor (same sublattice) pairs of sites. The homogeneous hopping amplitude $t$ in Eq. (11) is taken to be purely real; disorder appears in the perturbation $\delta t_{i, j}$. We take the amplitude $\delta t_{i, j}$ to be a Gaussian complex random variable with zero mean, statistically independent on different lattice links. The operators $\delta \hat{n}_{A / B} \equiv\left(c_{A / B}^{\dagger} c_{A / B}-\frac{1}{2}\right)$ in Eq. (11) denote the deviations of the local sublattice fermion densities from their value at half filling; the interaction strengths $V$ and $U$ appearing in this equation couple to nearest neighbor and next-nearest neighbor density-density interactions, respectively.

The Hamiltonian $H$ in Eq. (1) is invariant under the (antiunitary) sublattice symmetry (SLS) transformation

$$
c_{A i} \rightarrow c_{A i}^{\dagger}, \quad c_{B j} \rightarrow-c_{B j}^{\dagger},
$$

(all complex scalar terms in $H$ are complex conjugated) $\stackrel{19}{\underline{1}}$ In the clean limit, Eq. (11) with $\delta t_{i, j}=0$ for all lattice bonds $\langle i j\rangle$, SLS is responsible for the nesting condition of the noninteracting Fermi surface. As a result of nesting, the Fermi liquid phase of the clean model is unstable to charge density wave (CDW) order for any $2 V>U \geq 0 \stackrel{16,20}{=}$
The effects of the interparticle interactions $U$ and $V$ upon the delocalized phase of the disordered model given by Eq. (1) may be investigated by using Finkel'stein's generalized non-linear sigma model $(\mathrm{FNL} \sigma \mathrm{M})$ approach ${ }^{4.5}$ to formulate the low-energy effective continuum field theory. The latter can be studied using a controlled $\epsilon$ expansion in $d=2+\epsilon$ dimensions $(0 \leq \epsilon \ll 1)$. We use the Schwinger-Keldysh ${ }^{21}$ method to perform the ensemble average over realizations of the hopping disorder. The FNL $\sigma \mathrm{M}$ is derived following the standard methodology: ${ }^{4,5,21}$

In the present case, the resulting FNL $\sigma \mathrm{M}$ is described by the generating functional ${ }^{20}$

$$
Z=\int \mathcal{D} \hat{Q} e^{-S_{D}-S_{I}},
$$

where

$$
\begin{aligned}
S_{D}= & \frac{1}{8 \pi \lambda} \int d^{d} \mathbf{r} \operatorname{Tr}\left[\boldsymbol{\nabla} \hat{Q}^{\dagger}(\mathbf{r}) \cdot \nabla \hat{Q}(\mathbf{r})\right] \\
+ & h \int d^{d} \mathbf{r} \operatorname{Tr}\left\{i \hat{\Omega}\left[\hat{Q}^{\dagger}(\mathbf{r})+\hat{Q}(\mathbf{r})\right]\right\} \\
& -\frac{\lambda_{A}}{8 \pi \lambda^{2}} \int d^{d} \mathbf{r}\left\{\operatorname{Tr}\left[\hat{Q}^{\dagger}(\mathbf{r}) \boldsymbol{\nabla} \hat{Q}(\mathbf{r})\right]\right\}^{2},
\end{aligned}
$$

and

$$
\begin{aligned}
S_{I}= & i \sum_{a=1,2} \xi^{a} \int d t d^{d} \mathbf{r}\left\{2 \Gamma_{V} Q_{t t}^{\dagger a a}(\mathbf{r}) Q_{t t}^{a a}(\mathbf{r})\right. \\
& \left.+\Gamma_{U}\left[Q_{t t}^{a a}(\mathbf{r}) Q_{t t}^{a a}(\mathbf{r})+Q_{t t}^{\dagger a a}(\mathbf{r}) Q_{t t}^{\dagger a a}(\mathbf{r})\right]\right\} .
\end{aligned}
$$

The field variable

$$
\hat{Q}(\mathbf{r}) \rightarrow Q_{t t^{\prime}}^{a b}(\mathbf{r})
$$

in Eqs. (32)-(5) is a complex, "infinite-dimensional" square matrix living in $d$ spatial dimensions, where indices $t$ and $t^{\prime}$ belong to a continuous time or (via Fourier transform) frequency space, and where indices $a$ and $b$ belong to a 2-dimensional "Keldysh" species space, with $a, b \in\{1,2\}, 21,22$ In Eq. (4), Tr denotes a matrix trace over time (or frequency) and Keldysh indices. $\hat{Q}(\mathbf{r})$ satisfies in addition the unitary constraint

$$
\hat{Q}^{\dagger}(\mathbf{r}) \hat{Q}(\mathbf{r})=\hat{1} .
$$

The matrix $\hat{Q}$ and its adjoint $\hat{Q}^{\dagger}$ may be interpreted ${ }^{20}$ as continuum versions of the same-sublattice fermion bilinears

$$
\begin{aligned}
Q_{t t^{\prime}}^{a b} & \sim c_{A}^{a}(t) c_{A}^{\dagger b}\left(t^{\prime}\right), \\
Q_{t t^{\prime}}^{\dagger a b} & \sim c_{B}^{a}(t) c_{B}^{\dagger b}\left(t^{\prime}\right) .
\end{aligned}
$$

The action given by Eq. (4) describes the low-energy diffusive physics of the noninteracting random hopping model; a replica version of the noninteracting sigma model with action $S_{D}$ was originally studied by Gade and Wegner $\stackrel{3}{=}$ This noninteracting sector of the FNL $\sigma \mathrm{M}$ 
contains three coupling constants: $\lambda, \lambda_{A}$, and $h$. The parameter $1 / \lambda$ is proportional to the dimensionless $\mathrm{dc}$ conductance $g$ of the system (i.e., is related to the disorder strength), while $\lambda_{A}$ denotes a second measure of disorder, unique to this sublattice symmetry class, which strongly influences the single-particle density of states $\frac{3,10,11}{1}$ The parameter $\lambda_{A}$ may be simply interpreted as characterizing the strength of long-wavelength, quenched random orientational fluctuations in bond strength dimerization

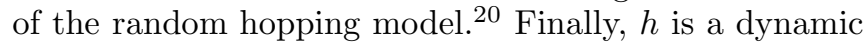
scale factor, which determines the dynamical critical exponent $z$ in Eq. (10e), below, through the condition $d \ln h / d l \equiv 0,5,13,20$ The interparticle interactions appear in $S_{I}$, defined by Eq. (5). Given Eq. (8), we may interpret $Q_{t t}^{a a}(\mathbf{r})$ and $Q_{t t}^{\dagger a a}(\mathbf{r})$ as continuum local density operators on the $A$ and $B$ sublattices, respectively. Then the interaction couplings $\Gamma_{V}$ and $\Gamma_{U}$ in Eq. (5) describe generic short-ranged intersublattice and same-sublattice densitydensity interactions, respectively [compare to Eq. (1), above]. Finally, $\xi^{a}= \pm 1$ in Eq. (5), for Keldysh species label $a=1,2$.

Using a Wilsonian frequency-momentum shell background field methodology $\underline{\underline{4}}$ we have performed a oneloop renormalization group calculation on the model defined by Eqs. (3)-(5). The calculation is straightforward, though rather lengthy; the details will be published elsewhere $\stackrel{20}{ }$ Below we simply state our results. In order to do so, it is convenient to introduce the following effective interaction couplings

$$
\gamma_{s} \equiv \frac{2}{\pi h}\left(\Gamma_{U}+\Gamma_{V}\right), \gamma_{c} \equiv \frac{2}{\pi h}\left(\Gamma_{U}-\Gamma_{V}\right) .
$$

The interaction strength $\gamma_{s}$ couples to the square of the (smooth) local charge density in the continuum theory, while $\gamma_{c}$ couples to the square of the sublattice staggered charge density. In accordance with the discussion in the paragraph below Eq. (2), we expect $\gamma_{c}<0$ to promote charge density wave (CDW) formation, while $\gamma_{c}>0$ should suppress it.

We find the following one-loop RG flow equations for the couplings $\lambda, \lambda_{A}, \gamma_{s}, \gamma_{c}$, and $h$ in $d=(2+\epsilon)$ dimensions:

$$
\begin{aligned}
\frac{d \lambda}{d l}= & -\epsilon \lambda-\lambda^{2} \gamma_{c} \\
& +2 \lambda^{2}\left[1+\frac{1-\gamma_{s}}{\gamma_{s}} \ln \left(1-\gamma_{s}\right)\right] \\
\frac{d \lambda_{A}}{d l}= & \epsilon \lambda_{A}+\lambda^{2}+2 \frac{\lambda_{A}}{\lambda} \frac{d \lambda}{d l} \\
\frac{d \gamma_{s}}{d l}= & \lambda_{A}\left(1-\gamma_{s}\right)\left(\gamma_{s}+2 \gamma_{c}-2 \gamma_{s} \gamma_{c}\right) \\
& -\lambda\left(1-\gamma_{s}\right)\left(\gamma_{s}+\gamma_{c}-2 \gamma_{s} \gamma_{c}\right) \\
\frac{d \gamma_{c}}{d l}= & \lambda_{A}\left(\gamma_{c}+2 \gamma_{s}\right)-\lambda\left(\gamma_{s}+\gamma_{c}\right) \\
& +\lambda\left[2 \gamma_{c} \ln \left(1-\gamma_{s}\right)+\gamma_{s} \gamma_{c}\right]-2 \gamma_{c}^{2} \\
\frac{d \ln h}{d l}= & (d-z)+\lambda_{A}+\lambda\left(\gamma_{c}-\gamma_{s}\right)
\end{aligned}
$$

Here, $l$ is the logarithm of the spatial length scale. These flow equations are given to the lowest non-trivial order in the couplings $\lambda, \lambda_{A}$, and $\gamma_{c}$, but contain contributions from $\gamma_{s}$ to all orders; Finkel'stein's NL $\sigma \mathrm{M}$ formulation provides 5 a perturbative expansion which is controlled by the (small) dimensionless resistance $\lambda$, but does not require the interaction strength $\gamma_{s}$ to be small. Before turning to an analysis of our results, Eqs. (10a)-(10e), we provide interpretations for various key terms appearing in them. First, the term in square brackets on the second line of Eq. (10a) is the usual correction to the dimensionless dc resistance $\lambda$, arising from the short-ranged interparticle interactions,,$\frac{4,23,24}{}$ and corresponds 25 to coherent backscattering of carriers off of disorder-induced Friedel oscillations in the background electronic charge density ${ }^{26}$ The last term in Eq. (10d) drives the CDW instability, which is a remnant of the clean Hubbard-like model (recall that in our conventions $\gamma_{c}<0$ signals this instability).

Now we analyze our results. In $d=2$ dimensions, integrating Eq. (10) for generic initial conditions shows that the critical, delocalized phase of the half-filled, noninteracting random hopping model $\underline{3}^{\underline{3}}$ is unstable to the effects of short-ranged interparticle interactions. We find that either $\gamma_{c} \rightarrow-\infty$ signaling CDW formation, or that $\lambda, \lambda_{A} \rightarrow \infty$ and $\gamma_{c} \rightarrow+\infty$, indicating a flow toward both strong disorder and strong interactions. Regardless, we expect the 2D interacting, disordered Hubbard model to be an insulator at zero temperature. This should be compared to an analogous result $\underline{\underline{13}}$ previously obtained for a TRI, interacting random hopping model on the honeycomb lattice. This physics is consistent with numerical studies $\frac{18}{18}$ of the half-filled spin- $1 / 2$ Hubbard model in $d=2$, which have shown that TRI random hopping disorder preserves the compressibility gap of the clean Mott insulator, and that the disordered and interacting system shows no signs of metallic behavior.

The situation in $d=(2+\epsilon)>2$ dimensions is more interesting. Upon increasing $\epsilon$ from zero, a narrow, irregularly shaped sliver corresponding to a stable metallic, diffusive Fermi liquid state opens up in the four-dimensional $\left(\lambda, \lambda_{A}, \gamma_{s}, \gamma_{c}\right)$ coupling constant space. The sliver encloses the line $\lambda=\lambda_{A}=\gamma_{c}=0$, with $-\infty<\gamma_{s}<1$, the entirety of which is perturbatively accessible because the FNL $\sigma \mathrm{M}$ does not require the interaction strength $\gamma_{s}$ to be small. A highly schematic 3D "projected" phase diagram is depicted in Fig. 1, In this figure, the interaction constants reside in the horizontal plane, while the vertical direction schematically represents (both) disorder strengths; the shaded sheath is a cartoon for the boundary of the stable metallic region, which resides between it and the ballistic $\left(\lambda=\lambda_{A}=0\right)$ plane. The "height" of the stable metallic region in the "disorder" directions $\left(\lambda, \lambda_{A}\right)$ is controlled by $\epsilon$, although the precise shape and size of the phase boundary varies with $\gamma_{s}$, and is difficult to characterize analytically. Over the range of perturbatively small values of $\gamma_{c}$, the stable Fermi liquid phase resides in the region $\gamma_{c} \gtrsim 0$, and terminates near $\gamma_{c}=0$. 


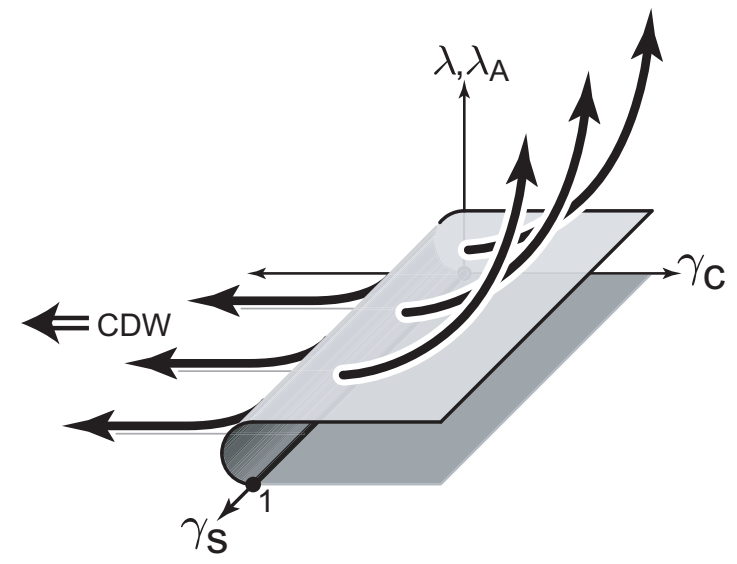

FIG. 1: Schematic phase diagram in $d=2+\epsilon$, with $0<\epsilon \ll 1$. $\lambda$ and $\lambda_{A}$ are measures of the disorder, whereas $\gamma_{s}$ and $\gamma_{c}$ characterize the interaction strengths. The stable metallic phase resides between the ballistic plane $\left(\lambda=\lambda_{A}=0\right)$ and the shaded sheath; the thick arrows indicate the two instabilities of the (metallic) diffusive Fermi liquid discussed in the text.

The flow equations (10a)-10d possess no perturbatively accessible, nontrivial RG fixed points for $d>2$, and thus no continuous metal-insulator transition can be identified. However, the two instabilities described above for the 2D case persist for $d>2$, and become clearly distinct roads out of the metallic state. The conventional CDW instability always occurs for initial $\gamma_{c}<0$ and sufficiently weak disorder, i.e., when $\lambda, \lambda_{A} \ll \epsilon$, and is represented by the flow $\gamma_{c} \rightarrow-\infty$. This flow is accompanied by a decay in both disorder strengths $\lambda, \lambda_{A}$.
The primary result of this paper is the identification of a second route out of the diffusive Fermi liquid phase in $d=(2+\epsilon)>2$ dimensions, independent of the Mott CDW instability, arising solely from the competition of disorder and interaction effects. As in the 2D case, this second route is characterized by a flow off to both strong disorder $\left(\lambda, \lambda_{A} \rightarrow \infty\right)$ and strong interactions $\left(\gamma_{c} \rightarrow+\infty\right)$, as indicated by the thick arrows emerging from the $\gamma_{c}>0$ portion of the phase boundary shown in Fig. 11 we call it an Anderson-Mott instability. Even though there is no perturbatively accessible fixed point, this new instability is nonetheless perturbatively controlled in $d=(2+\epsilon)$ over a wide range of initial conditions when $\epsilon \ll 1$; in particular, it is perturbatively accessible over the entire range $0 \leq \gamma_{s}<1.27$ Numerically integrating Eqs. (10a) - 10d) for small $\epsilon \ll 1$, we find that the Anderson-Mott instability can apparently always be reached by increasing only the dimensionless resistance $\lambda$. We expect the boundary separating the flow toward the stable metallic regime from that toward the regime of the Anderson-Mott instability to represent a disorder-driven, first order metal-insulator transition (MIT). We emphasize that a MIT does not exist in the noninteracting random hopping model, which possesses only a delocalized phase at half-filling for finite disorder in $d \geq 1,2,3,10,11$ while the clean spinless Hubbard model possesses only the Mott CDW instability. RG flow equations in related systems of spin-1/2 fermions were recently obtained independently in Ref. 28 .

This work was supported in part by the NSF under Grant No. DMR-00-75064 and by the UCSB Graduate Division (M.S.F.).
* Electronic address: psiborf@physics.ucsb.edu

1 For a review, see e.g. P. A. Lee, and T. V. Ramakrishnan, Rev. Mod. Phys. 57, 287 (1985).

${ }^{2}$ For recent references, see e.g.: L. Balents and M. P. A. Fisher, Phys. Rev. B 56, 12970 (1997); M. Bocquet, Nucl. Phys. B 546, 621 (1999).

3 R. Gade and F. Wegner, Nucl. Phys. B 360, 213 (1991); R. Gade, Nucl. Phys. B 398, 499 (1993).

4 A. M. Finkel'stein, Zh. Eksp. Teor. Fiz. 84, 168 (1983), Sov. Phys. JETP 57, 97 (1983).

5 For a review, see e.g. D. Belitz and T. R. Kirkpatrick, Rev. Mod. Phys. 66, 261 (1994).

${ }^{6}$ For a recent account see: S. V. Kravchenko and M. P. Sarachik, Metal-insulator transition in two-dimensional electron systems, Rep. Prog. Phys. 67, 1 (2004); A. Punnoose and A. M. Finkel'stein, Science 310, 289 (2005).

7 For a recent discussion, see e.g. A. Auerbach, Interacting Electrons and Quantum Magnetism (Springer-Verlag, New York, 1994).

8 M. R. Zirnbauer, J. Math. Phys. 37, 4986 (1996).

9 P. A. Lee and D. S. Fisher, Phys. Rev. Lett. 47, 882 (1981); A. Furusaki, Phys. Rev. Lett. 82, 604 (1998); M. Bocquet and J. T. Chalker, Phys. Rev. B 67, 054204 (2003); Ann. Henri Poincaré 4, S539 (2003).
10 S. Guruswamy, A. LeClair, and A. W. W. Ludwig, Nucl. Phys. B 583, 475 (2000).

11 M. Fabrizio and C. Castellani, Nucl. Phys. B 583, 542 (2000);

12 O. Motrunich, K. Damle, and D. A. Huse, Phys. Rev. B 65, 064206 (2002); C. Mudry, S. Ryu, and A. Furusaki, Phys. Rev. B 67, 064202 (2003).

13 M. S. Foster and A. W. W. Ludwig, Phys. Rev. B 73, 155104 (2006).

14 J. E. Hirsch, Phys. Rev. B 31, 4403 (1985).

15 J. E. Gubernatis, D. J. Scalapino, R. L. Sugar, W. D. Toussaint, Phys. Rev. B 32, 103 (1985).

16 R. Shankar, Rev. Mod. Phys. 66, 129 (1994).

17 Without interactions, the model is in class AIII of Ref. 8 .

18 M. Ulmke and R. T. Scalettar, Phys. Rev. B 55, 4149 (1997); P. J. H. Denteneer, R. T. Scalettar, N. Trivedi, Phys. Rev. Lett. 87, 146401 (2001).

19 In the presence of time-reversal invariance (TRI), SLS is equivalent to the usual particle-hole symmetry.

${ }^{20}$ M. S. Foster and A. W. W. Ludwig, (unpublished).

21 M. L. Horbach and G. Schoen, Ann. Phys. (Leipzig) 2, 51 (1993); A. Kamenev and A. Andreev, Phys. Rev. B 60, 2218 (1999); C. Chamon, A. W. W. Ludwig, and C. Nayak, Phys. Rev. B 60, 2239 (1999). 
22 The symbol $\hat{\Omega}$ appearing in Eq. (4) denotes a familiar matrix (Refs. 20.21) diagonal in frequency (and Keldysh) indices.

${ }^{23}$ B. L. Altshuler and A. G. Aronov, in Electron-Electron Interactions in Disordered Systems, edited by A. L. Efros and M. Pollak (North-Holland, Amsterdam, 1985.)

${ }^{24}$ E. P. Nakhmedov, V. Prigodin, S. Çalışkan, E. Şaşıog̃lu, Phys. Rev. B 66, 233105 (2002).

${ }^{25}$ A. M. Rudin, I. L. Aleiner, and L. I. Glazman, Phys. Rev.
B 55, 9322 (1997); I. L. Aleiner, B. L. Altshuler, and M. E. Gershenson, Waves Random Media 9, 201 (1999).

26 Background density fluctuations become a source of onsite disorder in the presence of electron-electron interactions, so that we may attribute this nontrivial correction to "dynamic SLS breaking."

27 We do not consider here $\gamma_{s}<0$.

28 L. Dell'Anna, Nucl. Phys. B 758, 255 (2006). 